Horizontes. Revista de Investigación en Ciencias de la Educación

ISSN 2616-7964

abril-junio, 2017

HORIZONTES

Volumen 1, Número 2

www.revistahorizontes.org

pp. $11-22$

\title{
El significado del paro universitario - caso Universidad Central de Venezuela
}

\section{The meaning of university unemployment - case Central University of Venezuela}

\author{
Luisa Margarita París Lara \\ parisluisi@gmail.com \\ Universidad Central de Venezuela (UCV)
}

Recibido: 10-10-2016 / Revisado: 13-10-2016 / Aceptado: 14-11-2016 / Publicado: 10-04-2017

\begin{abstract}
RESUMEN
Este trabajo se orientó en abordar el significado del paro universitario como acción por parte de algunos integrantes de la comunidad ucevista, para alcanzar sus reivindicaciones salariales. El objetivo de esta investigación fue comprender el significado del paro, que tienen integrantes de esta institución educativa (profesores, estudiantes, obreros y empleados administrativos). Se utilizaron dos enfoques teóricos: el interaccionismo simbólico y la teoría crítica. El trabajo fue de corte cualitativo. Se aplicaron entrevistas en profundidad a miembros de las Facultad de Ciencias Jurídicas y Políticas y de la Facultad de Humanidades y Educación de la Universidad Central de Venezuela. Para el procesamiento, análisis e interpretación de los datos se utilizó el análisis de contenido. Como resultado los informantes revelaron no estar muy a favor del paro, pero a su vez consideran que este permite logros reivindicativos en lo salarial.
\end{abstract}

Palabras clave: Paro, Significado, Reivindicación Salarial, Comunidad universitaria, Participación
This work focused on addressing the meaning of university unemployment as an action by some members of the Uzbek community to meet their salary demands. The objective of this research was to understand the meaning of unemployment, which have members of this educational institution (teachers, students, workers and administrative employees). Two theoretical approaches were used: symbolic interactionism and critical theory. The work was a qualitative one. In-depth interviews were applied to members of the Faculty of Legal and Political Sciences and the Faculty of Humanities and Education of the Central University of Venezuela. For the processing, analysis and interpretation of the data we used the content analysis. As a result, the informants revealed that they were not very in favor of unemployment, but in turn considered that this allows for wage claims.

Key words: Unemployment, Meaning, Wage Claim, University community, Participation 


\section{INTRODUCCIÓN}

El presente trabajo de investigación se centra en la exploración del significado del paro universitario como acción para lograr las reivindicaciones salariales por parte del sector universitario, en específico de dos facultades de la Universidad Central de Venezuela (UCV). En este sentido se plantea explorar el significado que tiene el paro para algunos de los integrantes de la comunidad universitaria: profesores, estudiantes, obreros y empleados administrativos.

Para tal fin se recurre a dos enfoques teóricos tales como: el interaccionismo simbólico, enfoque que trabaja con la significación social y la teoría crítica de Ignacio Martín Baró, bajo la figura del influjo interpersonal.

El marco metodológico que le da forma a la investigación, es cualitativo, dado que es una realidad presentada cara a cara a la investigadora, de las voces de sus protagonistas, en específico de las facultades de Humanidades y Educación, y Ciencias Jurídicas y Políticas. Se destaca que se escogieron dieciséis (16) informantes claves, tomando en cuenta a cuatro (4) informantes por cada sector trabajado. La técnica utilizada para recolectar los datos es la entrevista en profundidad, como método de análisis se emplea el análisis de contenido que permite organizar los datos en categorías y subcategorías, a fin de dar cuenta del fenómeno estudiado. Se analizan los resultados derivados de cada entrevista, y se da la discusión de los mismos, centrados en las convergencias y divergencias presentadas por los informantes en relación con el significado del paro universitario como reivindicación salarial.

\section{Una aproximación al paro universitario desde una mirada psicosocial}

"La Universidad Central de Venezuela (UCV) fue fundada en el año 1721, desde su creación la Universidad Central de
Venezuela es núcleo importante del desarrollo de la vida intelectual venezolana; su papel en el país es esencial y constituye una referencia fundamental para la comprensión de los procesos de transformación de la sociedad venezolana." (Brioli, 2012, p.1)".

En esencia, la Universidad Central de Venezuela es una de las instituciones de educación superior más antiguas del país. Sin embargo, hay ciertas situaciones que se le afectan, una de ellas asociada al problema presupuestario que a su vez redunda en lo salarial y desencadenan una acción específica: el paro, cuyo significado es el punto focal de este trabajo.

Para tener un referente aproximado a la actualidad, se toma en cuenta el siguiente acontecimiento del año 2013 (publicado por Salcedo Flores, en la página del noticiero Venevisión, que evidencia la realidad nacional universitaria y aspectos que conducen al paro específicamente el día10/06/2013.

“(...) La Universidad Central de Venezuela inició este lunes formalmente un paro indefinido de actividades luego de la petición que hacen de aumento de sueldo y salarios para profesores, trabajadores, obreros y personal administrativo (...) Todo el sector universitario tiene peticiones: sueldos, aumento en las becas, mejoras en la infraestructura (...) Dicen que el Estado ha enviado beneficios a las universidades. (...) Guillermo Guerrero del sindicato obrero de la UCV indica que 'esperemos el señor ministro Calzadilla, ministro de esta casa de estudios que se nos ofrezca un salario digno sepa la comunidad en general que los trabajadores universitarios no poseemos aumento salarial 2011, 2012,2013' (...)".

Se justifica el paro bajo una situación que afecta a la comunidad universitaria, como se indica en la cita anterior. En consecuencia, hablar del paro como esa acción que desarrolla el sector universitario ante la 
inconformidad frente al problema salarial, aunado al significado que pueda tener para los actores sociales que participan en el mismo, lo convierte en objeto para la investigación psicosocial.

La Universidad Central de Venezuela (UCV), a simple vista puede describirse como una micro sociedad, en la cual converge multiplicidad de actores sociales cuya participación activa le da movilidad a la referida institución educativa. Estos actores que confluyen en el escenario universitario son diversos, desde profesores, estudiantes, obreros, personal administrativo, y la interacción de los mismos le da permanencia en tiempo y espacio.

El interés particular en abordar el paro universitario, deriva de la necesidad de conocer cuál es el universo de significados de algunos actores sociales que forman parte de la dinámica universitaria involucrado e indagar qué efecto puede generar en lo interno (estrés, angustia, incertidumbre).

Para tal fin se recurre a dos enfoques teóricos: El interaccionismo simbólico que trabaja con la interpretación de los significados sociales que los seres humanos le dan a la conducta a partir de la construcción de los mismos y las acciones que pueden ser generadas por ellos, dentro de los distintos ámbitos de interacción: el paro es una acción generada por algunos actores sociales de la comunidad ucevista como respuesta a una situación particular, en la búsqueda hacia el logro de una reivindicación salarial.

“(...) el ser humano orienta sus actos hacia las cosas en función de lo que éstas significan para él (...) nos referimos a todo aquello que una persona puede percibir en su mundo: objetos físicos; como árboles o sillas; otras personas, como una madre o un dependiente de comercio; categorías de seres humanos, como amigos o enemigos; instituciones, como una escuela o un gobierno; ideales importantes, como la independencia individual o la honradez; actividades ajenas, como las órdenes o peticiones de los demás; y las situaciones de todo tipo que un individuo afronta en su vida cotidiana(...)" (Blumer, 1982, p. 2).

Otro elemento teórico es el punto del influjo interpersonal trabajado en la teoría crítica de Ignacio Martin Baró, donde se señala que el ser humano actúa en solitario y de otro modo en colectivo, dado que los demás pueden tener influencia. Lo anterior aplicado al plano real puede indicar que una cosa es lo que el actor piense o el significado que le otorgue al paro y otra es si se hace partícipe o no en el mismo. Por otra parte, como es la relación con el paro en sí mismo.

“(...) el influjo de la presencia de los otros pasa por el filtro del propio individuo, que cae en la cuenta de esa presencia. Por otro lado, la conciencia siempre es una conciencia de algo; los otros presentes tienen una significación para el sujeto, quien valora positiva o negativamente esa presencia y anticipa las consecuencias buenas o malas que le puede acarrear. Así, la presencia de otras personas pondrá nervioso al individuo o le dejará tranquilo, le estimulará o le será indiferente, le agradará o le molestará. (Baró, 1990, p.14)".

Lógicamente, el paro no actúa por sí solo, se nueve por los actores involucrados en el mismo al presentarse esta situación, la cual redunda en la paralización de las actividades educativas que conlleva a reacciones ante lo mismo.

\section{Abordaje metodológico}

Este trabajo de investigación trata directamente a la realidad tomada desde las voces de sus protagonistas (de los cuáles se ha hecho mención en párrafos anteriores). En consecuencia, esta investigación realizada tiene un carácter cualitativo.

La investigación se desarrolla en el campus universitario, los informantes seleccionados hacen vida activa en la Universidad Central de Venezuela (UCV), en 
específico pertenecen a las Facultades de Humanidades y Educación, y Ciencias Jurídicas y Políticas. Se seleccionaron dieciséis (16) informantes claves, proporcionales a los cuatro sectores (se distribuyen cuatro informantes por cada sector). Los mismos fueron seleccionados bajo el muestreo intencional.

Según Ruiz (2009):

"El muestreo intencional es aquel en el que los sujetos de la muestra no son elegidos siguiendo las leyes del azar sino de una forma intencional. En él no hay modo de estimar la probabilidad de que cada elemento tiene que ser incluido en la muestra (...)" (p.64).

De acuerdo a las características de la investigación ya expuestas, el método para la producción de información más preciso es la entrevista a profundidad. Se realizaron cuatro (4) entrevistas por representante de cada sector para dar un total de dieciséis (16 entrevistas), a fin de conocer los distintos criterios en torno al tema de investigación desarrollado. Se destaca que para cerrar la recolección de datos se utilizó el criterio de saturación de la muestra.

Carolina Martínez Salgado (2011) cita a Morse para dar una definición precisa del criterio de saturación:

"El precepto exige recolectar datos hasta que ocurra la saturación. Pero ¿Qué significa saturación? ¿Cuáles son sus fundamentos y sus principios? En el ámbito de la investigación cualitativa se entiende por saturación el punto en el cual se ha escuchado ya una cierta diversidad de ideas $y$ con cada entrevista $\mathrm{u}$ observación adicional no aparecen ya otros elementos".

El análisis de los datos se realizó bajo el análisis de contenido, del cual se despendieron una serie de categorías y subcategorías enmarcados en ciertas disensiones que dan cuenta del fenómeno estudiado (véase cuadros resumen por sector).

\section{Discusión de los datos}

De acuerdo a los resultados arrojados, el paro es esa acción única en la cual confluyen estos actores con sus acuerdos, desacuerdos, pero de una u otra forma son los sectores estudiados quienes hacen vida alrededor del paro y se influyen por el fenómeno indicado.

Hay una convergencia de criterios en referencia a la génesis del paro por parte de los sectores docente, estudiantil, obrero y trabajador administrativo, respecto a la existencia de una crisis universitaria, la cual redunda en la falta y fallas en el presupuesto, reflejado en la carencia de recursos para el funcionamiento de la universidad, que repercute en el desarrollo de la actividad docente y la formación de los futuros profesionales.

Los docentes y estudiantes coinciden en un reconocimiento de que la labor formativa generada en las aulas de clase por la parte profesoral, tiene un carácter vocacional. Sobre todo, en ese contexto de inconstantes aumentos salariales en el cual está inmersa la universidad y la presencia del paro como medio para subsanar esa situación presentada a lo largo de esta investigación.

El sector estudiantil enfatiza su necesidad de expresarse ante la situación de la universidad, como parte de la comunidad universitaria, para tal fin es preciso hacerse sentir desde las aulas, al convertirlas en espacios para la discusión, desde sus áreas de formación profesional. Dado que sus aportes desde sus áreas de formación son importantes para generar buenas propuestas que trabajen en pro de la solución en lo interno de los males que aquejan a la Universidad Central de Venezuela (UCV).

Para los entrevistados de los cuatro sectores existe un hecho evidente, como lo es la división entre la parte docente, obrero y administrativa a pesar de ser protagonistas de una realidad que les afecta (en este caso bajos salarios). Al momento de ejecutar un paro para reclamar sus aumentos, cada quien está 
en su espacio. Si se trata de actores que pertenecen a un mismo escenario, como la Universidad Central de Venezuela, lo ideal es lograr la unificación de criterios y de sectores, poder participar en caso de realizarse un paro, para así hacer más contundente ese reclamo de salarios mejores, que a la práctica es un mal común de los trabajadores de la universidad en sus distintas áreas la docencia y la labor obrera o administrativa.

Un aspecto tocado unívocamente por el sector obrero, es el papel que desempeña el sindicato como el ente que se encarga de organizar el paro y a la vez presionar para que los objetivos que se plantean sean cubiertos, en consecuencia, alcanzar las reivindicaciones salariales.

Respecto al tema salarial los docentes enfatizan en el problema de la universidad enmarcado en un hecho latente: los bajos salarios y el presupuesto en esa misma condición son parte del reflejo de la realidad país, la falta de recursos afecta el desempeño del docente es su praxis investigativa y laboral. Los docentes plantean con inquietud la poca importancia que se le da a la universidad a pesar de ser la principal casa de estudios de Venezuela, no se le da la relevancia que debería tener. El mismo punto desde la óptica de los trabajadores administrativos redunda en la disparidad salarial en relación con la economía nacional aunado a la canasta básica, lo cual indica que el dinero ganado no alcanza.

Los estudiantes expresan que como parte de la crisis del sector universitario la educación ha bajado de calidad porque los docentes se han enfocado en hacer paros y hay un reconocimiento de que la universidad trabaja con las uñas, a pesar de la situación que atraviesa.

Del paro en sí hay múltiples significados que permiten dar varias miradas a un mismo fenómeno, en función de puntos en común o disensos desde las voces de sus protagonistas que han servido como informantes clave, que sirven de punto de partida para revelar todo aquello que pueda surgir desde sus propias percepciones en su interacción con el paro universitario.

El sector docente y administrativo converge en el paro como una medida de presión que actúa como cable conductor a un logro reivindicativo salarial para aquellos integrantes de la comunidad universitaria que le dan movilidad laboral. Se define como ese mecanismo de reclamo utilizado por excelencia dentro del entorno universitario.

Los docentes se refieren al paro como parte del descontento ante la situación universitaria sobre todo la salarial, a su vez al momento de plantearlo como acción no hay organización y falta de motivación e igualmente es un atraso para el sector universitario en su totalidad.

Desde el sector estudiantil surgen ópticas muy interesantes desde ver al paro como boicot al sistema educativo universitario, que perjudica la formación académica de los profesionales, a su vez se constituye en un limbo académico cargado de incertidumbre en torno al destino de las actividades en aula y continuidad de los semestres. El paro tiene un componente político traducido en divergencias entre las partes laborales, y en otro sentido tampoco el paro significa la solución a los problemas de la universidad, que a medida que se han desglosado de los relatos han sido presentados.

Para los obreros y trabajadores administrativos la última opción a la cual se recurre es el paro, producto de demandas no satisfechas. Otro aspecto también es ir de paro y no obtener a la larga ese salario acorde con la realidad nacional.

Los representantes de los cuatro sectores entrevistados convergen en el hecho de que el paro no es del todo eficaz como se plantea, los alcances en salarios acorde con la realidad no son del todo completos, sin embargo, esto puede tener mayor sentido al enfatizar en el efecto que ejerce el paro en los actores que 
protagonizan la realidad universitaria, tomados como informantes claves.

Los cuatro sectores entrevistados convergen en que el paro repercute de una manera negativa, este redunda en atraso tanto en el desarrollo de las actividades laborales del sector administrativo y obrero, igualmente interfiere en el desenvolvimiento de las labores académicas desempeñadas por los profesores, lo cual a su vez afecta a la formación estudiantil, dado que el paralizar las actividades docentes implica un retardo en el desarrollo y culminación del semestre.

El estudiantado al afirmar experimentar atraso en el semestre y la posible pérdida del mismo, manifiestan un temor cada vez que escuchan la palabra paro, lo cual le da un componente emocional al efecto del mismo. No están de acuerdo con este por lo ya planteado.

Del sector obrero se destaca que hay una resistencia al paro en el sentido de expresar que no permite el desarrollo de las actividades con normalidad y a su vez no permite estar en el espacio universitario, el cual es ideal para hacer esos reclamos reivindicativos, en materia de salario al cual se ha hecho referencia a lo largo de este trabajo de investigación.

Profesores, estudiantes y personal administrativo convergen en la idea de que el paro es desmotivador, sobre todo al momento de darle ejecución a sus actividades cotidianas (dictar clases, formación académica y labores administrativas. En tanto se evidencia como el paro afecta negativamente, no solo desde las prácticas académico - laborales y formativas sino también en lo emocional.

Retomando el efecto del paro, este no es del todo negativo, tal como se demostró anteriormente, para el sector administrativo es un logro reivindicativo porque gracias al mismo han conseguido pagos de aumentos salariales que redundan en beneficio para los trabajadores.
Por otra parte, para el personal administrativo el paro es beneficioso, pero a la vez es perjudicial, esto recoge lo anteriormente señalado, en relación con el esperado aumento salarial y un efecto negativo extendido a la totalidad de las actividades propias de la dinámica universitaria tales como académicas, docentes, administrativas y obreras.

El sector docente recalca que muchos colegas aprovechan del uso del ocio y el tiempo libre en tiempos de paro, lo cual le da un carácter de vacación al mismo.

Los docentes y estudiantes convergen en la idea de que existen diferencias políticas en algunos de los integrantes de la comunidad universitaria, sobre todo aquellos que hacen vida laboral, por tanto, en medio de esa necesidad de alcanzar equilibrios salariales es necesario establecer unificación ente los gremios.

En el caso de los docentes, se ve al paro como una opción conductora a los beneficios demandados por ellos (aumento salarial), pero debe complementarse con actividades de calle que den a conocer la situación del sector universitario.

Si bien es cierto, el paro se percibe como un elemento que lleva al logro de las reivindicaciones salariales para algunos de los integrantes de la comunidad ucevista, de acuerdo al criterio de los informantes pueden haber alternativas al paro, parte de esa mirada enfocada en la no efectividad del todo del paro, unido a esa repercusión negativa del mismo resumida en atraso en las actividades, posibles pérdidas de semestre más ese componente emocional desmotivador cargado de incertidumbre, sobre todo para los estudiantes.

Como alternativa al paro, el sector docente propone realizar campañas informativas fuera del espacio universitario a fin de dar a conocer qué sucede en la máxima casa de estudios, igualmente la universidad debe tener una participación activa en las 
luchas salariales. En otro sentido para poder conocer a profundidad cuál es la situación real de la universidad, lo más idóneo es realizar un diagnóstico de las necesidades por facultad a razón de construir ese escenario bajo el cual se diseñarán las estrategias para solicitar los requerimientos necesarios para abordar la situación universitaria.

Docentes, obreros y personal administrativo coinciden en establecer mesas de trabajo, con el objeto de abordar los distintos puntos neurálgicos que pueda presentar el sector universitario, es una forma de estar activos en el campus sin abandonarlo.

El sector estudiantil expresa que en lugar de mesas de trabajo es mejor utilizar la figura de la mesa de diálogo, en la cual se planteen distintos puntos de vista y aspectos de la situación de la universidad, acompañado de una comisión de personas destinadas a ejecutar el diálogo. En la misma línea, propone que todos los sectores deberían abrir espacios para el diálogo con la finalidad de intercambiar ideas e idear estrategias que los beneficien, dado que, si se trata del tema salarial, este afecta a la totalidad de la población laboral de la Universidad Central de Venezuela y es preciso tratarlo en conjunto, no aislado, donde cada quien esté por su lado.

Para el sector obrero, dialogar con el gobierno es primordial dado que es el agente que dota de recursos económicos a la universidad y es una forma de plantear esas necesidades de los trabajadores universitarios, igualmente, las acciones de calle pueden ser efectivas (marchas) punto en el cual se converge con los trabajadores administrativos.

En general, el paro tiene una carga muy significativa para los actores involucrados, a pesar de los consensos y disensos que se tejen a su alrededor, es considerado el camino para alcanzar las esperadas reivindicaciones salariales, al mismo tiempo forma parte de la cotidianidad de algunos integrantes de la comunidad ucevista, no es un fenómeno desconocido, el grado de conocimiento y familiaridad parte de todos los sectores, docentes, estudiantes, obreros y trabajadores administrativo.

Se puede decir que paro es sinónimo de reclamo y descontento por parte del personal laboral universitario, por una situación salarial con la no se está conforme y es preciso solventar, esta es la acción generada para llegar al camino de las reivindicaciones salariales.

Si bien es cierto, el sector laboral ucevista ya sea en sus prácticas docentes, obreras o administrativas se afectan por los bajos salarios e igualmente el paro repercute en su desempeño laboral. El sector más vulnerable, puede decirse que está en el medio de la paralización de las actividades dentro del campus universitario es el estudiantil, dado que el retraso en sus clases repercute en la culminación a tiempo de sus cargas académicas.

Hablar del paro universitario convoca a la existencia de que el funcionamiento de la Universidad Central de Venezuela, no está del todo bien, lo ideal sería que esta realidad cambiara y el paro pasara a ser parte de una opción extrema de última hora, pero dadas las circunstancias se constituye en parte del entorno universitario $\mathrm{y}$ de aquellos integrantes que hacen vida activa en la misma y son los motores de su funcionamiento, ya sean profesores, estudiantes, obreros 0 personal administrativo. 


\section{Cuadros resumen por sector}

\section{Cuadro 1. Profesores}

\begin{tabular}{|c|c|c|c|}
\hline Dimensión & Categoría & & Sub-categoría \\
\hline \multirow{7}{*}{$\begin{array}{l}\text { Situación del sector } \\
\text { universitario }\end{array}$} & \multirow{5}{*}{ Génesis del paro } & 1 & Crisis interna \\
\hline & & 2 & Realidad país \\
\hline & & 3 & Falta de recursos \\
\hline & & 4 & Bajos salarios docentes \\
\hline & & 5 & $\begin{array}{l}\text { Comprensión de la importancia de } \\
\text { la UCV }\end{array}$ \\
\hline & Vocación de servicio & 6 & Compromiso institucional \\
\hline & Desunión de sectores & 7 & Necesidad de unificación \\
\hline \multirow{13}{*}{ l paro } & \multirow{4}{*}{ Significado del paro } & 8 & $\begin{array}{l}\text { Paro como instrumento de } \\
\text { presión }\end{array}$ \\
\hline & & 9 & Descontento \\
\hline & & 10 & Medida de presión \\
\hline & & 11 & Desorganización \\
\hline & & 12 & Necesidad de motivación \\
\hline & \multirow{6}{*}{ Efecto del paro } & 13 & Sin eficacia \\
\hline & & 14 & $\begin{array}{l}\text { Pérdida de tiempo para las } \\
\text { actividades académicas }\end{array}$ \\
\hline & & 15 & Paralización de la investigación \\
\hline & & 16 & Estudiantes perjudicados \\
\hline & & 17 & Inasistencia a clase \\
\hline & & 18 & Paro como vacación \\
\hline & $\begin{array}{c}\text { A pesar de las diferencias } \\
\text { políticas } \\
\end{array}$ & 19 & Unificación gremial \\
\hline & El paro como opción & 20 & Actividades complementarias \\
\hline \multirow{4}{*}{ Otra opción al paro } & \multirow{4}{*}{ Alternativa al paro } & 21 & Campaña informativa \\
\hline & & 22 & Participación activa UCV \\
\hline & & 23 & Mesas de trabajo \\
\hline & & 24 & $\begin{array}{l}\text { Diagnóstico de necesidades por } \\
\text { facultad }\end{array}$ \\
\hline
\end{tabular}




\section{Cuadro 2. Estudiantes}

\begin{tabular}{|c|c|c|c|}
\hline Dimensión & Categoría & & Sub-categoría \\
\hline \multirow{9}{*}{$\begin{array}{l}\text { Situación del sector } \\
\text { universitario }\end{array}$} & \multirow{7}{*}{ Génesis del paro } & 1 & Crisis general y polarización \\
\hline & & 2 & $\begin{array}{l}\text { Baja calidad de la educación } \\
\text { universitaria }\end{array}$ \\
\hline & & 3 & Necesidades sector estudiantil \\
\hline & & 4 & Problema presupuestario \\
\hline & & 5 & Dependencia del Estado \\
\hline & & 6 & La universidad trabaja con las uñas \\
\hline & & 7 & Desatención a la Universidad \\
\hline & $\begin{array}{c}\text { Abordaje de la realidad en } \\
\text { las aulas }\end{array}$ & 8 & Opinión del estudiantado \\
\hline & Vocación de servicio & 9 & Compromiso institucional \\
\hline \multirow{12}{*}{ El paro } & \multirow{5}{*}{ Significado del paro } & 10 & Paro como boicot \\
\hline & & 11 & Limbo académico \\
\hline & & 12 & Participación estudiantil \\
\hline & & 13 & Diferencias políticas \\
\hline & & 14 & No es la solución \\
\hline & \multirow{6}{*}{ Efecto del paro } & 15 & Desmotivación \\
\hline & & 16 & Atraso académico \\
\hline & & 17 & No viable \\
\hline & & 18 & Atraso \\
\hline & & 19 & Temor al paro \\
\hline & & 20 & Perdida de semestre \\
\hline & $\begin{array}{c}\text { A pesar de las diferencias } \\
\text { políticas }\end{array}$ & 21 & Unidad gremial \\
\hline \multirow{4}{*}{ Otra opción al paro } & \multirow{4}{*}{ Alternativa al paro } & 22 & $\begin{array}{l}\text { Espacios de diálogo entre todos los } \\
\text { sectores }\end{array}$ \\
\hline & & 23 & Necesidad de una auditoría interna \\
\hline & & 24 & Mesas de dialogo \\
\hline & & 25 & Comisión para el dialogo \\
\hline
\end{tabular}




\section{Cuadro 3. Obreros}

\begin{tabular}{|c|c|c|c|}
\hline Dimensión & Categoría & & Sub-categoría \\
\hline \multirow{8}{*}{$\begin{array}{l}\text { Situación del sector } \\
\text { universitario }\end{array}$} & \multirow{4}{*}{ Génesis del paro } & 1 & $\begin{array}{l}\text { Intereses políticos de las } \\
\text { autoridades }\end{array}$ \\
\hline & & 2 & Descuido a la universidad \\
\hline & & 3 & $\begin{array}{l}\text { Falta de dotación uniformes sector } \\
\text { obrero }\end{array}$ \\
\hline & & 4 & Renovación de autoridades \\
\hline & Papel del sindicato & 5 & Ejercer presión \\
\hline & $\begin{array}{c}\text { Lucha por } \\
\text { reivindicaciones } \\
\text { estudiantiles (Comedor, } \\
\text { transporte e } \\
\text { infraestructura) }\end{array}$ & 6 & Papel de estudiantado \\
\hline & \multirow{2}{*}{ División de sectores } & 7 & $\begin{array}{l}\text { Necesidad de unificación de } \\
\text { sectores }\end{array}$ \\
\hline & & 8 & Lucha salarial de todos los sectores \\
\hline \multirow{9}{*}{ El paro } & \multirow{3}{*}{ Significado del paro } & 9 & No paralizar labores \\
\hline & & 10 & Pérdida de sentido del paro \\
\hline & & 11 & Paro como último recurso \\
\hline & \multirow{5}{*}{ Efecto del paro } & 12 & Paro no efectivo \\
\hline & & 13 & Realización de labores normal \\
\hline & & 14 & Atraso laboral y académico \\
\hline & & 15 & Logro reivindicativo \\
\hline & & 16 & No permite estar en la UCV \\
\hline & Participación & 17 & Apatía \\
\hline \multirow{5}{*}{ Otra opción al paro } & \multirow{4}{*}{ Alternativa al paro } & 18 & Mesas de trabajo \\
\hline & & 19 & Dialogo con el gobierno \\
\hline & & 20 & $\begin{array}{l}\text { Necesidad de unificación de } \\
\text { sectores }\end{array}$ \\
\hline & & 21 & Acciones de calle \\
\hline & Paro como única opción & 22 & Mecanismo presión reforzado \\
\hline
\end{tabular}


Cuadro 4. Empleados administrativos

\begin{tabular}{|c|c|c|c|}
\hline Dimensión & Categoría & & Sub-categoría \\
\hline \multirow{8}{*}{$\begin{array}{l}\text { Situación del sector } \\
\text { universitario }\end{array}$} & \multirow{7}{*}{ Génesis del paro } & 1 & Salario no acorde con la cesta básica \\
\hline & & 2 & $\begin{array}{l}\text { No reconocimiento de las peticiones } \\
\text { del sector universitario }\end{array}$ \\
\hline & & 3 & Ajuste salarial \\
\hline & & 4 & Falta de presupuesto \\
\hline & & 5 & Problema presupuestario \\
\hline & & 6 & Descontento con la situación laboral \\
\hline & & 7 & Sectores no tomados en cuenta \\
\hline & División de sectores & 8 & Indiferencia entre sectores \\
\hline \multirow{9}{*}{ El paro } & \multirow{2}{*}{ Significado del paro } & 9 & Medida de presión \\
\hline & & 10 & Última opción \\
\hline & \multirow{6}{*}{ Efecto del paro } & 11 & Paro beneficioso y perjudicial \\
\hline & & 12 & Poca efectividad \\
\hline & & 13 & Perdida de clases \\
\hline & & 14 & Paro perjudicial \\
\hline & & 15 & Afecta emocionalmente \\
\hline & & 16 & Afecta el desempeño laboral \\
\hline & Participación & 17 & Apoyo de todos los sectores \\
\hline \multirow{5}{*}{ Otra opción al paro } & \multirow{5}{*}{ Alternativa al paro } & 18 & Mesas de trabajo \\
\hline & & 19 & Lucha de calle \\
\hline & & 20 & Lucha en el espacio universitario \\
\hline & & 21 & Negociación \\
\hline & & 22 & Dialogo \\
\hline
\end{tabular}

CONCLUSIÓN

Haber abordado el significado del paro universitario como objeto de investigación psicosocial ha sido enriquecedor, solo por el hecho de ver como los actores de la comunidad universitaria profesores, estudiantes, obreros y trabajadores administrativos, revelan cómo es su relación con ese fenómeno que ya forma parte de su cotidianidad, dado que al hablar de aumentos salariales le sugiere automáticamente al paro como acción para lograr ese fin reivindicativo. El paro por sí mismo deriva de una situación que afecta al sector universitario, en este caso se particularizó en la Universidad Central de Venezuela (UCV) que se encuentra inmersa en un escenario de inconsistentes aumentos salariales, alejados de la realidad económica nacional.
Del paro se tejen multiplicidad de significados:

Se ve al paro como ese recurso que permite alcanzar las reivindicaciones salariales, dado que en cierto modo da frutos, a pesar de ser considerado como no efectivo. Ya estar en paro se ve como algo normal en la comunidad ucevista, sin embargo, a criterio de los cuatro sectores informantes tomados en cuenta, tiene un efecto perjudicial dado que conlleva al atraso en el desarrollo de las actividades académicas, igualmente sucede con las labores del personal obrero $\mathrm{y}$ administrativo.

En otro sentido se habla de un sector vulnerable a juicio de los profesores, obreros y administrativos: los estudiantes, quienes reciben el efecto directo del paro con las inconsistencias en los inicios y continuidad de 
los semestres. La población estudiantil ve al paro como un ente que los lleva a un limbo académico, cargado de incertidumbres, desmotivación, tiene un componente emocional.

Por otro lado, para el personal administrativo el paro desmotiva, ante la incertidumbre de lograr ese aumento esperado y a la vez genera un bajo desempeño laboral.

La comunidad universitaria lo ve como esa medida de presión producto de la inconformidad ante lo salarial, igualmente es la última opción a la cual se recurre en caso de no ver las demandas salariales satisfechas.

De acuerdo a lo explorado y plasmado a largo de este trabajo de investigación, el paro universitario en tiempos de crisis ha tenido una permanencia y continuidad dentro del espacio universitario, de la mano de sus protagonistas.

Blumer H (1982) El interaccionismo simbólico. Perspectiva y método. Barcelona, España.

Brioli, C. (2012) Breve reseña histórica de la Universidad Central de Venezuela Recuperado de: http://ucvnoticias.ucv.ve/wordpress/?pag e_id $=429$.

Fuenmayor, L., (2004) El Financiamiento Universitario. Revista Foro Universitario, Educere saber.ula.ve (26), pp. 385-395.

Martín Baró, I. (1990) Acción e ideología. Psicología Social desde Centroamérica. San Salvador, San Salvador: UCA editores.

Martínez C. (2011). El muestreo en investigación cualitativa. Principios básicos $\mathrm{y}$ algunas controversias. Recuperado de: http://www.scielosp.org/pdf/csc/v17n3/v 17n3a06.pdf

Ruiz, José (2009). Metodología de la investigación cualitativa. Bilbao, España: Universidad de Deusto. 